Simon Collin

Université du Québec à Montréal (Canada)

Jean Gabin Ntebutse

Université de Sherbrooke (Canada)

\title{
Introduction au dossier
}

\section{Les théories critiques et le numérique en éducation : quelles propositions théoriques et quelles applications empiriques?}

Les théories critiques constituent un ensemble hétérogène de courants de recherche (traditions marxistes, École de Francfort, gender studies, black studies, études postcoloniales, etc.), qui n'a cessé d'évoluer en fonction des changements sociaux qu'elles étudient (Keucheyan, 2013). Bien qu'elles se caractérisent autant par leurs ressemblances que par leurs différences, il est possible de les résumer comme une posture scientifique à la fois explicative, normative et pratique (Bohman, 2016; De Munck, 2011), qui permet d'appréhender et d'orienter les enjeux de domination et d'émancipation entre individus et groupes sociaux. Interdisciplinaires, elles disposent de traditions bien établies dans certaines disciplines, alors qu'elles sont plus discrètes dans d'autres. En sciences de l'éducation au Québec, les théories critiques ont principalement trouvé leur marque dans les «fondements » de l'éducation (p. ex., philosophie et sociologie de l'éducation). En revanche, elles ont moins été exploitées en psychopédagogie ou en didactique, lesquelles prédominent dans l'étude du numérique en éducation. Il en découle que ce dernier a peu été abordé en termes critiques. Ce constat n’est pas spécifique au Québec et vaut pour l'espace tant francophone (Collin, Guichon et Ntebutse, 2015) qu'anglophone (Selwyn, 2015). Plus précisément, les théories critiques ont été appliquées au cas du numérique en éducation à partir des années 1980, avec des auteurs tels que Cuban, Apple et Noble aux États-Unis. Elles ont été sporadiquement mobilisées par la suite, de manière parfois implicite et hétérogène, sans constituer une tradition pour autant.

Toutefois, depuis les années 2010, le domaine du numérique en éducation semble connaître un regain d'intérêt pour les théories critiques, qui se manifeste par l'organisation d'événements scientifiques et de projets de publication qui leur sont consacrés, à l'instar de ce numéro thématique. On peut y voir un effort de structuration en cours, qui est susceptible de contribuer grandement à l'avancement des connaissances dans le domaine. En effet, à l'heure où les GAFA (Google, Amazon, Facebook, Apple) de ce monde s'avèrent aussi non intéressées qu'inaptes à gérer les implications sociales qu'elles produisent; à l'heure aussi où les mêmes 
GAFA investissent de plus en plus le « marché » éducatif, il y a tout lieu de penser que les théories critiques ont toute leur place dans le domaine du numérique en éducation. En dépassant les enjeux pragmatiques de la salle de classe, elles permettent d'en couvrir d'autres - critiques - connexes à l'enseignement et à l'apprentissage per se.

Ce numéro thématique souhaite contribuer au travail de structuration des théories critiques sur le numérique en éducation. Il réunit à la fois des articles théoriques (en première partie du numéro), qui réfléchissent aux tenants et aboutissants des théories critiques pour l'étude du numérique en éducation, et des articles empiriques (en deuxième partie du numéro), qui adoptent une perspective critique dans leur étude du numérique en éducation.

Le premier article, celui de Neil Selwyn (Monash University, Australie), propose un panorama exhaustif des théories critiques du numérique en éducation dans l'espace anglophone. Cet article est précieux à plus d'un titre : d'abord, parce qu'il mobilise la littérature anglophone, qui est de loin la plus fournie et la plus dynamique sur cette question; ensuite, parce que l'aperçu qu'il propose conjugue rétrospection et prospection; enfin, parce que Neil Selwyn est le principal leader, à l'échelle internationale, des théories critiques du numérique en éducation, ce qu'il doit à la finesse et à la perspicacité de ses analyses.

Le deuxième article est rédigé par Périne Brotcorne (Université catholique de Louvain, Belgique), Simon Collin (Université du Québec à Montréal, Canada) et Élisabeth Schneider (Université de Caen, France) et propose un regard très original sur les théories critiques du numérique en éducation. Jouant sur leur nature résolument interdisciplinaire, il en dresse tour à tour un portrait en sciences humaines et sociales de manière générale, puis en sciences de l'éducation, puis dans le domaine du numérique en éducation. Cette lecture "en cascade " permet d'avancer que le domaine du numérique en éducation n'a pas pleinement exploité, jusqu'à maintenant, la richesse des travaux critiques présents en sciences de l'éducation et en sciences humaines et sociales plus généralement. C'est donc tout autant une invitation à poursuivre les efforts de structuration en cours.

Le troisième article, celui de Julie Denouël (Université Rennes 2, France), propose un prolongement très pertinent des bases théoriques jetées par Collin et al. (2015). En partant du constat que l'approche sociocritique développée par ces auteurs se focalise principalement sur le cas des apprenants, l'article propose de l'appliquer au cas des enseignants, en en précisant du même coup certains aspects. Pour ce faire, l'article mobilise un appareillage théorique arrimant les théories critiques et les approches sociotechniques au moyen des concepts de double médiation sociotechnique, d'appropriation, de capital technique et de reconnaissance.

Le quatrième article, rédigé par Carine Aillerie (Université de Poitiers, France), souligne avec pertinence la contribution de la notion de translittératie à l'approche sociocritique proposée par Collin et al. (2015) et aux théories critiques du numérique en éducation de manière générale. Après avoir défini cette notion, l'article l'opérationnalise dans le cadre d'une recherche empirique, en rendant compte des usages numériques de lycéens français (deuxième moitié du secondaire, au Québec). La translittératie s'offre ainsi comme une voie théorique permettant d'appréhender les usages numériques des acteurs éducatifs dans la complexité de leur triple dimension médiatique, sociale et contextuelle.

Le cinquième article est proposé par Simon Collin (Université du Québec à Montréal, Canada), Valerie Steeves (Université d'Ottawa, Canada), Jacquie Burkell (Western University, Canada) et Yannick Skelling-Desmeules (Université du Québec à Montréal, Canada). Il s'agit d'une recherche

4 - Formation et profession 27(3), 2019 
empirique dont l'origine réside dans la question fondamentale suivante : l'école contribue-t-elle à réduire ou à reproduire les inégalités numériques présentes chez les jeunes en contexte extrascolaire? La méthodologie repose sur une analyse secondaire d'une base de données pancanadienne délèves de 9 à 16 ans. Les résultats indiquent que le niveau socioéconomique des élèves est lié à des différences marginales mais significatives en termes d'accès et d'usage numériques à l'école et en dehors, alors que la médiation enseignante ne varie pas.

Le sixième article, celui de Jean Horvais (Université du Québec à Montréal, Canada), aborde la question très pertinente et trop peu documentée de l'éducation aux médias d'élèves adolescents ayant une déficience intellectuelle ou un trouble du spectre de l'autisme (DI/TSA). Plus précisément, l'article présente une étude empirique qualitative réalisée auprès d'enseignants en adaptation scolaire, dans le but de mieux comprendre leurs perceptions et leurs intentions à l'égard de l'éducation aux médias de leurs élèves. Sujet crucial, car il pose la question, d'une part, de l'accessibilité des populations en situation de handicap au numérique, et, d'autre part, des risques de stigmatisation qui peuvent découler d'usages principalement compensatoires visant à soutenir cette accessibilité. Sur ce point, l'article témoigne d'un positionnement avisé des enseignants à l'égard des médias numériques, capables de discerner avec réalisme à quelles conditions ces derniers peuvent soutenir la participation scolaire et sociale de leurs élèves.

Enfin,le septième article, rédigé par Jean-François Ceci (Université de Pau et des Pays de l'Adour, France), propose une caractérisation empirique de « l'apprenant numérique » sur la base d'un échantillon d'élèves et d'étudiants allant du collège français (début du secondaire, au Québec) à la maitrise universitaire. Sur la base d'analyses qualitatives et quantitatives, les résultats indiquent que, derrière l'hyperconnexion des élèves et des étudiants, ces derniers entretiennent un rapport ambigu et variable au numérique à l'école. En effet, l'apprenant numérique se caractérise notamment par une forte sensation d'apprentissage avec le numérique, mais principalement de manière informelle et en contexte extrascolaire, ce qui redouble la question de l'articulation entre école et numérique.

En somme, les articles de ce numéro thématique abordent les théories critiques du numérique en éducation sous des angles riches et variés, ce qui est à l'image de l'hétérogénéité des théories critiques et de leur état de développement dans le domaine du numérique en éducation.

\section{Références}

Bohman, J. (2016). Critical theory. Dans E. N. Zalta (dir.), The Stanford Encyclopedia of Philosophy. Repéré à https://plato. stanford.edu/archives/fall2016/entries/critical-theory/

Collin, S., Guichon, N. et Ntebutse, J. G. (2015). Une approche sociocritique des usages numériques en éducation. STICEF, (22), 89-117. http://dx.doi.org/10.3406/stice.2015.1688

De Munck, J. (2011). Les trois dimensions de la sociologie critique. SociologieS. Repéré à http://sociologies.revues.org/3576

Keucheyan, R. (2013). Hémisphère gauche : une cartographie des nouvelles pensées critiques. Paris : La Découverte.

Selwyn, N. (2015). Technology and education: Why it's crucial to be critical? Dans S. Bulfin, N. Johnson et C. Bigum (dir.), Critical perspectives on technology and education (p. 245-255). New York, NY : Palgrave Macmillan. http://dx.doi.org/10.10 $\underline{57 / 978113738545114}$

\section{Pour citer cet article}

Collin, S. et Ntebutse, J. G. (2019). Introduction du dossier : Les théories critiques et le numérique en éducation: quelles propositions théoriques et quelles applications empiriques? Formation et profession, 27(3), 3-5. http://dx.doi.org/10.18162/fp.2019.578 